Algebraic $K$-Theory 


\section{Mathematics and Its Applications}

Managing Editor:

M. HAZEWINKEL

Centre for Mathematics and Computer Science, Amsterdam, The Netherlands

Volume 311 


\section{Algebraic $K$-Theory}

by

Hvedri Inassaridze

Georgian Academy of Sciences,

Tbilisi, Georgia

Springer-Science+Business Media, B.V. 
Library of Congress Cataloging-in-Publication Data

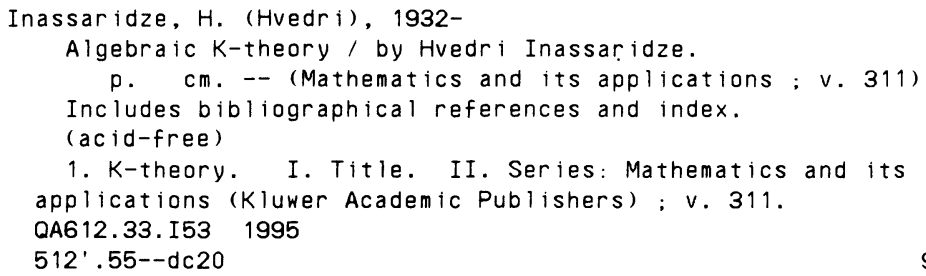

Printed on acid-free paper

All Rights Reserved

ISBN 978-90-481-4479-2 ISBN 978-94-015-8569-9 (eBook)

DOI 10.1007/978-94-015-8569-9

(C) 1995 Springer Science+Business Media Dordrecht

Originally published by Kluwer Academic Publishers in 1995.

Softcover reprint of the hardcover 1st edition 1995

No part of the material protected by this copyright notice may be reproduced or utilized in any form or by any means, electronic or mechanical, including photocopying, recording or by any information storage and retrieval system, without written permission from the copyright owner. 
To Indo Inassari 


\section{Contents}

$\begin{array}{ll}\text { Introduction } & 1\end{array}$

Chapter I. Classical Algebraic K-functors 3

$\S$ 1. The Grothendieck functor $K_{0} \quad 3$

A. Definition, examples and some properties 3

B. $\operatorname{Spec}(\mathrm{R})$ and $\mathrm{H}(\mathrm{R}) \quad 12$

C. Serre's theorem 18

§ 2. The Bass-Whitehead functor $K_{1}^{B} \quad 29$

$\S 3$. The Milnor functor $K_{2}^{M} \quad 35$

Chapter II. Higher $K$-functors 43

$\S 1$. $K$-theory of Quillen for exact categories 43

§ 2. The Quillen plus construction $\quad 72$

A. Definition and properties $\quad 72$

B. Computation of $K_{*}^{Q}(k)$ for a finite field $k$

C. Quillen's $K$-groups for free algebras $\quad 96$

D. Negative algebraic $K$-theory 102

E. Finite generation of Quillen's $K$-groups of the rings of algebraic integers 108

F. Transfer map in the localization theorem 117

§ 3. $K$-Theory of Swan 127

$\S 4$. $K$-theory of Karoubi-Villamayor $\quad 140$

$\S 5$. $K$-theory of Waldhausen 149

Chapter III. Properties of algebraic K-functors 163 
$\S 1$. Exactness, excision and the Mayer-Vietoris sequence

A. Exactness

B. Excision

C. The Mayer-Vietoris sequence

$\S 2$. The localization theorem

$\S 3$. The fundamental theorem

$\S 4$. Products in algebraic $K$-theory

Chapter IV. Relations between algebraic $K$-theories

$\S 1$. Isomorphism of Quillen's algebraic $K$-theories. Agreement of plus construction and $Q$-construction

$\S 2$. Connection of Quillen's plus construction with Swan's algebraic $K$-theory

$\S 3$. Comparison of Swan's and Karoubi-Villamayor's algebraic $K$ theories

Chapter V. Relation between algebraic and topological $K$-theories 289

$\S 1$. Equivalence of categories of finitely generated projective modules and vector bundles over a compact space for $C^{*}$-algebras

$\S 2$. $K$-theory of special normed algebras and $\mathbb{Z}_{2}$-graded $C^{*}$-algebras 305

A. $K$-theory of special normed algebras 305

B. $K$-theory of $\mathbb{Z}_{2}$-graded $C^{*}$-algebras 328

$\S 3$. Isomorphism of Swan's and Karoubi-Villamayor's $K$-theories with topological $K$-theory for real Banach algebras

Chapter VI. The problem of Serre for polynomial and monoid algebras

$\S 1$. Proof of Anderson's conjecture

A. Normal and seminormal monoids

B. Projective modules over normal monoid rings

C. On the triviality of the Picard group for monoid algebras over a principal ideal domain

$\S 2$. The algebraic proof of Swan

Chapter VII. Connection with cyclic homology 